\title{
PLANETARY NEBULAE IN GALAXIES BEYOND THE LOCAL GROUP
}

\author{
H. C. Ford and R. Ciardullo, \\ Space Telescope Science Institute \\ Homewood Campus, Baltimore, MD 21218 \\ G. H. Jacoby \\ Kitt Peak National Observatory, Tucson, AZ 85726 \\ X. Hui \\ Boston University, Boston, MA 02215
}

\begin{abstract}
.
Planetary nebulae can be used to estimate the distances to galaxies and to measure stellar dynamics in faint halos. We discuss surveys which have netted a total of 665 candidate planetary nebulae in NGC 5128 (Cen A), NGC 5102, NGC 3031 (M81), NGC 3115, three galaxies in the Leo Group (NGC 3379, NGC 3384, NGC 3377), NGC 5866, and finally, in NGC 4486 (M87). Radial velocities of planetaries in M32 have shown that its halo velocity dispersion is most likely isotropic. Radial velocities of planetaries in M31 show that $\sim 2 / 3$ of the nebulae with projected radii between 15 and $30 \mathrm{kpc}$ are members of a rotating thick disk with slight asymmetric drift, while $\sim 1 / 3$ belong to a slowly rotating halo. Velocities of 116 nebulae in NGC 5128 reveal pronounced rotation and a slowly declining velocity dispersion in the halo out to $20 \mathrm{kpc}$. The [O III] $\lambda 5007$ luminosity functions (PNLFs) in NGC 5128, M81, and the three Leo Galaxies have the same shape over the first magnitude. The highly consistent distances derived from the brightnesses of the $j^{\text {th }}$ nebula and the median nebula in different fields in the same galaxy and from different galaxies in the same group lend strong support to the suggestion that planetaries are an accurate standard candle in old stellar populations. Comparison of theoretical luminosity functions to the observed PNLFs shows that there is a very small dispersion in the central star masses.
\end{abstract}

\section{INTRODUCTION}

There are at least three reasons to search for planetary nebulae (PN) in galaxies beyond the Local Group. First, they are the only easily resolvable component in old stellar populations at large distances. The detection of PN provides a direct measure of the stellar death rate, mass return rate, and flux of ionizing radiation in an old stellar population. Second, $\mathrm{PN}$ are readily identified test particles whose radial velocities can be used to measure the mass distribution, mass-to-light ratios, 
and stellar dynamics in the innermost and outermost regions of early type galaxies. As a probe of galactic halos, they are complementary to, and, in many respects, superior to globular clusters. In galaxies which are closer than the Virgo cluster, there are far more detectable planetaries than globular clusters, and, with an onband/off-band interference filter technique, they are much easier to find. Planetary nebulae provide one of the few, if not only, means of measuring the dynamics of the faint shells found around some peculiar galaxies. The third reason for seeking planetaries in distant galaxies is the possibility that the luminosities of the brightest PN may be a good standard candle in early type galaxies and old populations such as spiral bulges. This paper will review a continuing long term program which emphasizes the latter two points. Detailed results will be given for the galaxy NGC 5128 .

\section{PLANETARY NEBULAE IN GALAXIES FROM HERE TO THE VIRGO CLUSTER}

Detection of faint nebulae in distant galaxies requires long integrations with a 4-m class telescope on dark nights with good seeing. The high quantum efficiency and dynamic range of a CCD camera are prerequisites for success. The nebulae are found by blinking an image taken through a narrow band interference filter which transmits a redshifted emission line against a continuum image taken through an interference filter which transmits an adjacent, emission line free region of the spectrum. Luminous high excitation planetaries are most easily detected in the light of [O III] $\lambda 5007$ for several reasons: 1) [O III] $\lambda 5007$ is usually the brightest optical line, 2) the contrast between [O III] $\lambda 5007$ and the sky plus galaxy continuum is better than any other strong optical lines, and 3) thinned CCDs have high quantum efficiency near $\lambda 5007$. A filter bandpass (FWHM) between 30 and $40 \AA$ optimizes detection of the nebulae with a single filter. If the bandpass is much narrower than $30 \AA$ the velocity dispersion in a giant elliptical galaxy will shift the observed wavelengths of many nebulae onto the shoulders of the filter. If the bandpass is much wider than $40 \AA$, the signal-to-noise ratio (SNR) is lowered. A practical consideration is the fact that the fast focal ratio ( $\mathrm{f} / 2.77)$ at the prime focus of the CTIO and KPNO 4-m telescopes will broaden the bandpass of very narrow band filtors to between 20 and $30 \AA$ and will lower the peak transmission (Eather and Reasoner, 1969). Care also must be taken when ordering narrow band filters to allow for bandpass shifts due to the ambient temperature (usually a few $\AA$ to the blue) and a fast beam (10 to $12 \AA$ to the blue at f/2.77). The bandpass of the off-band filter can be between 200 and $300 \AA$ wide in order to shorten the continuum exposure time. The off-band image should be at least 0.25 magnitudes deeper than the on-band image to avoid spurious detections at the noise limit.

Our total on-band exposure times range from one to six hours per field. Our identification criteria for planetary nebulae are: 1) appearance in each on-band frame and in the sum of the on-band frames, 2) absence in each off-band frame and in the sum of the off-band frames, 3) a point source profile, and 4) in the NGC 5128 and M81 fields where we took $\mathrm{H} \alpha$ images, an $\mathrm{H} \alpha$ to [O III] $\lambda 5007$ excitation ratio less than 0.9. The latter two criteria help discriminate against supernova remnants 
and against $H$ II regions in later type galaxies. We are confident that the majority of our candidates are bona fide planetaries because we find them in more than one frame and derive [O III] $\lambda 5007$ fluxes which are consistent with planetaries at the estimated distance of the galaxy. In NGC 5128 we independently verified the reality of 116 planetaries by measuring their radial velocities.

The results of our recent surveys for planetary nebulae are summarized in Table 1. The last column in the table gives the total number of candidate planetary nebulae in each galaxy.

Table 1. [O III] $\lambda 5007$ On-band/Off-band Survey for Planetary Nebulae

\begin{tabular}{|c|c|c|c|c|c|c|}
\hline $\begin{array}{l}\text { Galaxy } \\
(\mathrm{NGC})\end{array}$ & $\begin{array}{l}\text { Type } \\
\text { (RSA) }\end{array}$ & $\left(\begin{array}{c}v_{o b s} \\
\left.\mathrm{~km} \mathrm{~s}^{-1}\right)\end{array}\right.$ & $\begin{array}{c}\text { Fields } \\
\text { Surveyed }\end{array}$ & $\begin{array}{l}\text { Images } \\
\text { per Field }\end{array}$ & $\begin{array}{c}\text { Exposure Time } \\
(\mathrm{min})\end{array}$ & $\begin{array}{l}\text { Number of } \\
\text { Candidates }\end{array}$ \\
\hline 5128 & $\mathrm{~S} 0+\mathrm{S}_{\text {pec }}$ & 526 & 29 & 1 & 60 & 222 \\
\hline 5102 & $\mathrm{SO}_{1}(5)$ & 420 & 3 & 1 & 60 & 27 \\
\hline 3031 & $\mathrm{Sb}(\mathrm{r}) \mathrm{I}-\mathrm{II}$ & -36 & 1 & 3 & 30 & 185 \\
\hline 3115 & $\mathrm{SO}_{1}(7)$ & 655 & 2 & 2 & 90 & 68 \\
\hline 5866 & $\mathrm{SO}_{3}(8)$ & 672 & 1 & 3 & 60 & 18 \\
\hline 4486 & E0 & 1254 & 1 & 6 & 60 & 12 \\
\hline \multicolumn{7}{|c|}{ Leo Group } \\
\hline 3379 & E0 & 893 & 2 & 1 & 90 & 48 \\
\hline 3384 & $\mathrm{SBO}_{1}(5)$ & 771 & 2 & 1 & 90 & 53 \\
\hline 3377 & E6 & 718 & 1 & 1 & 90 & 32 \\
\hline
\end{tabular}

We have taken $\mathrm{H} \alpha$ on-band/off-band images of five galaxies in Table 1 to search for novae. The $\mathrm{H} \alpha$ filters were 65 to $75 \AA$ wide, which maximizes the SNR for expanding nova shells but reduces the detectability of planetaries and $\mathrm{H}$ II regions. Nonetheless, the $\mathrm{H} \alpha$ images can be used to help separate the brightest high excitation planetary nebulae from lower excitation $\mathrm{H}$ II regions and supernova remnants. Details of the $\mathrm{H} \alpha$ survey are given in Table 2. The six fields in NGC 5128 were surveyed once each in 1985, 1986, and 1987.

Table 2. $\mathrm{H} \alpha$ On-band/Off-band (Nova) Survey

\begin{tabular}{lccc}
\hline $\begin{array}{c}\text { Galaxy } \\
(\text { NGC) }\end{array}$ & Fields Surveyed & $\begin{array}{c}\text { Number of Images } \\
\text { per Field }\end{array}$ & $\begin{array}{c}\text { Exposure Time per Image } \\
\text { (min) }\end{array}$ \\
\hline 5128 & 6 & 4 & 45 \\
3031 & 1 & 7 & 10 \\
3115 & 1 & 9 & 10 \\
5866 & 1 & 2 & 45 \\
4486 & 1 & 3 & 60 \\
\hline
\end{tabular}

Figure 1 shows the positions of the 2 envelope fields (107 planetary nebulae) and 27 halo fields (115 planetary nebulae) in NGC 5128. The halo fields were 
chosen near the major axis of the faint outer isophotes (Dufour et al. 1978) and on both sides of the galaxy in order to measure rotation in the halo.

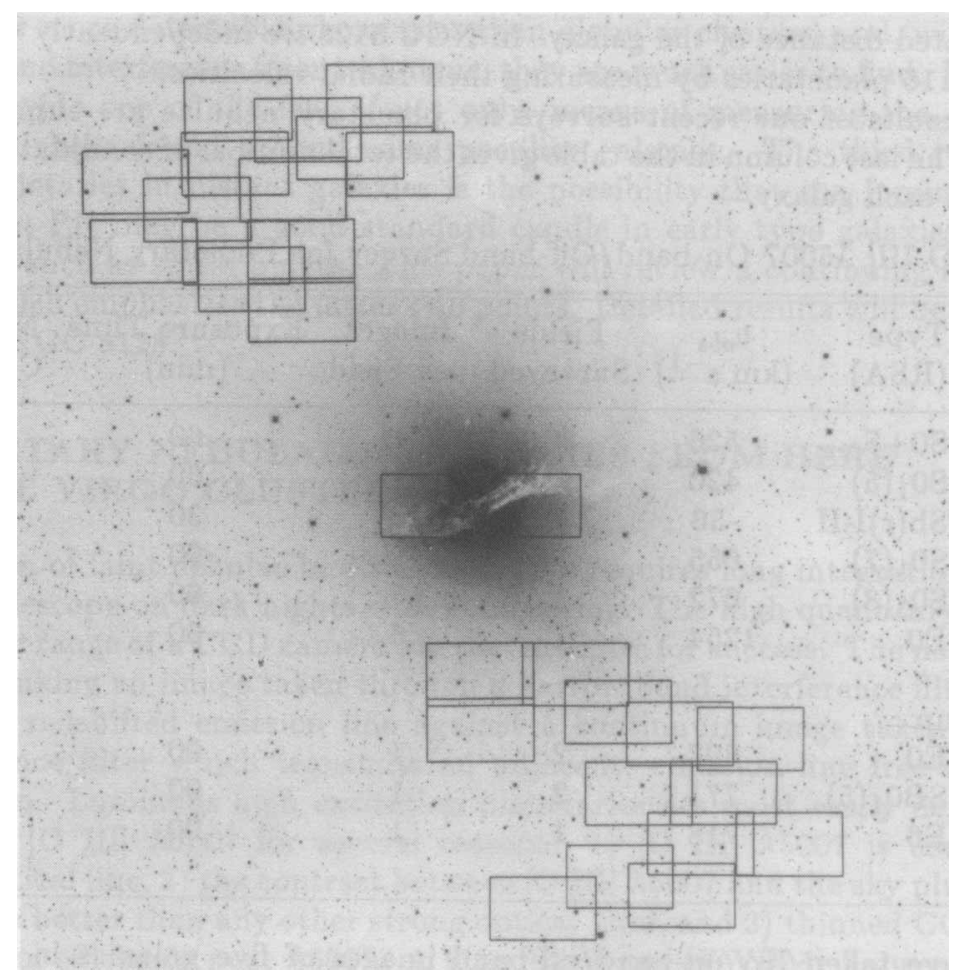

Figure 1. Each rectangle shows a CCD survey field projected onto NGC 5128. The dimensions of the CCD fields are $5^{\prime} \times 3^{\prime}(4.8 \mathrm{kpc} \times 2.9 \mathrm{kpc}$ at $3.3 \mathrm{Mpc})$. The centers of the most distant fields are $\sim 20 \mathrm{kpc}$ from the nucleus.

The number of PN per unit light in the NGC 5128 halo fields is shown in Figure 2. Because of seeing differences between the fields, the PN were counted to a constant [O III] $\lambda 5007$ magnitude which was well above the detection limit in all of the images. The two envelope fields were excluded because of the continuously varying limiting magnitude and the likelihood that the western field is partially veiled by dust in the warped plane which projects into the famous dust lane. The blue light in each field was calculated by numerically integrating an analytical model which incorporates Dufour et al.'s (1979) $r^{1 / 4}$ law along the major axis and the observed changing ellipticity of the isophotes. The number of planetaries per unit light appears constant throughout the halo at a value of 1.14 planetaries per unit light ( 1 unit for $\left.m_{B}=15\right)$. Numerical integration across the entire galaxy gives $m_{B}=7.48$ for the elliptical component, a value which should be more accurate than Dufour et al.'s value of 7.14 which was derived by assuming the isophotes are spherical. The estimated total number of planetary nebulae to our flux limit (which spans 1.6 magnitudes of the luminosity function) is then 1161 


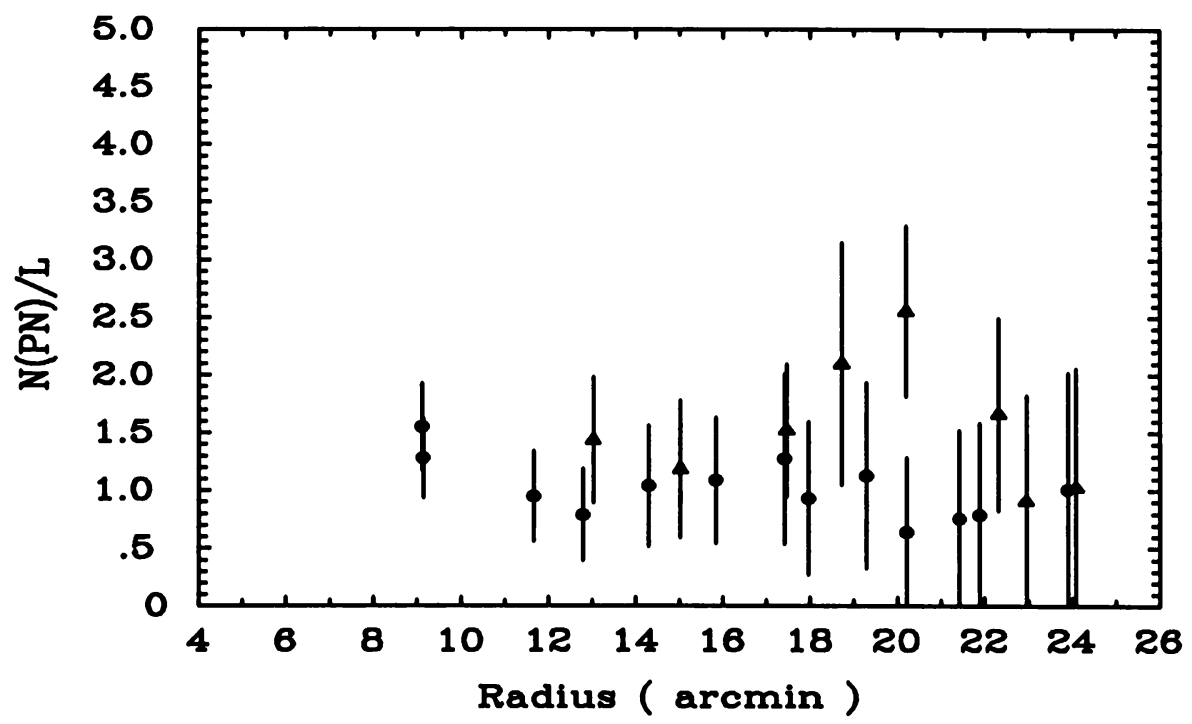

Figure 2. The number of planetary nebulae per unit light versus distance from the center of the galaxy. The filled circles are fields in the NE halo and the filled triangles are fields in the SW halo.

nebulae.

\section{PLANETARY NEBULAE AND STELLAR DYNAMICS}

Planetary nebulae have been used to measure the stellar dynamics in the LMC (Dopita et al., 1988 ), M32 (Nolthenius 1984; Nolthenius and Ford, 1986 [NF86]), M31 (Nolthenius 1984; Nolthenius and Ford 1987 [NF87]), NGC 205 (Jacoby and Ford 1988) and NGC 5128 (Ford et al. 1988). This review will not consider the LMC.

\subsection{M32 and NGC 205}

NF86 investigated velocity anisotropy in the halo of M32 and derived M32's mass and $\mathrm{M} / \mathrm{L}$ by analyzing the radial velocities of 15 halo planetaries. Because the sample was too small for binning, Nolthenius (1984; cf NF86) developed a procedure for testing the goodness of fit between small numbers of radial velocities and trial velocity dispersions derived from models. Spherical models with constant anisotropy (Jaffe 1983) and with radially varying anisotropy (Merritt 1985) were combined with the observed nuclear velocity dispersion (Tonry 1984; Whitmore 1980) to derive the trial velocity dispersions. NF86's most important conclusion is that the stellar velocities are close to isotropic throughout M32's halo. The rms dispersion of the planetaries is $42 \mathrm{~km} \mathrm{~s}^{-1}$, sufficiently lower than the nuclear dispersion of $70-80 \mathrm{~km} \mathrm{~s}^{-1}$ to make a strongly increasing $\mathrm{M} / \mathrm{L}$ with radius seem unlikely. Finally, the most likely fits give a mass of $8.2 \pm 2 \times 10^{8} M_{\odot}$ and a $\mathrm{M} / \mathrm{L}_{B}$ 
of 3-4 in solar units.

Jacoby and Ford (1988) have measured the radial velocities of 10 planetaries in NGC 205. The rms velocity dispersion is $27 \mathrm{~km} \mathrm{~s}^{-1}$ about the $-219 \mathrm{~km} \mathrm{~s}^{-1}$ mean heliocentric velocity.

\subsection{M31}

Lawrie and Ford (1982) used a Velocity Modulating Camera to observe 45 planetaries within $200 \mathrm{pc}$ of the center of M31. Lawrie (1983) measured the radial velocities for 33 of the nebulae to derive a systemic velocity of $-309 \pm 25$ $\mathrm{km} \mathrm{s}^{-1}$ and a velocity dispersion of $156 \pm 23 \mathrm{~km} \mathrm{~s}^{-1}$. His velocity dispersion is equal to the mean (156 $\left.\pm 9 \mathrm{~km} \mathrm{~s}^{-1}\right)$ of the velocity dispersion estimates for M31's bulge which were derived from automated analysis techniques (see Lawrie 1983 for references). Lawrie found no evidence to support the claim that there may be systematic or subtle biases which cause the Fourier methods to overestimate the velocity dispersion in early-type galaxies.

Nolthenius (1984) and NF87 used the radial velocities of 34 planetaries with projected distances between 15 and $30 \mathrm{kpc}$ to separate the nebulae into disk and halo populations. Approximately $1 / 3$ of the planetaries are members of a slowly rotating halo whose $92 \pm 43 \mathrm{~km} \mathrm{~s}^{-1}$ rotational velocity and $116 \pm 48 \mathrm{~km} \mathrm{~s}^{-1}$ velocity dispersion are very similar to the $80 \pm 28 \mathrm{~km} \mathrm{~s}^{-1}$ rotational velocity and $130 \mathrm{~km} \mathrm{~s}^{-1}$ velocity dispersion of the globular clusters (Huchra et al. 1982). Jacoby and Ford (1986) measured chemical abundances in two halo planetaries, one at a projected distance of $33 \mathrm{kpc}$ and the other at $3.5 \mathrm{kpc}$. The distant halo nebula has a low oxygen abundance which is between the $O$ abundance of the galactic halo planetaries $49+88.1$ and $K 648$, whereas the high velocity nebula at $3.5 \mathrm{kpc}$ has $\mathrm{O}, \mathrm{N}$, and $\mathrm{Ne}$ abundances between the Orion nebula and galactic planetaries. The M31 globular clusters have a wide range of metallicity and show little, if any, correlation between kinematics and integrated line strengths (Huchra et al. 1982; van den Bergh 1969). The abundances and especially the kinematics of the halo planetaries suggest that they derive from a population similar to that of the globular clusters.

The planetaries in the outer disk $(R \geq 15 \mathrm{kpc})$ are rotating a little more slowly $\left(14 \mathrm{~km} \mathrm{~s}^{-1}\right)$ than the gas in the disk, a value which is comparable to the observed asymmetric drift of galactic planetaries (Cahn and Wyatt 1978; Cudworth 1974). The derived azimuthal velocity dispersion is $\sigma_{\theta}=38 \pm 12 \mathrm{~km} \mathrm{~s}^{-1}$, and the inferred scale height is $z_{0}=1$ to $3 \mathrm{kpc}$, implying that the planetaries are part of a thick disk.

\subsection{NGC 5128}

We have begun a program in collaboration with $\mathrm{K}$. Freeman and M. Dopita to study the halos of early-type galaxies, and have chosen NGC 5128 (Cen A), the nearest giant elliptical and luminous radio source, as the first galaxy for our investigation. In this review we report a preliminary analysis of the radial velocities of 116 planetary nebulae found in the envelope and remote halo of Cen $A$. The radial velocities and a detailed comparison of the velocities to models of Cen 
A will be published elsewhere (Ford et al. 1988). The spectra of the nebulae were obtained during two nights in the spring of 1987 by using the multifiber spectrograph on the AAT. The velocities for individual PN were measured from the [O III] $\lambda 5007$ line, which had between 50 to 300 photon counts. The estimated accuracy of the measurement varies from $10 \mathrm{~km} \mathrm{~s}^{-1}$ for nebulae with 50 to 100 counts to about $4 \mathrm{~km} \mathrm{~s}^{-1}$ for PN with 100 to 200 counts.

The velocities of the planetary nebulae show indisputably that the halo is rotating ( $\mathrm{NE}$ approaching, $\mathrm{SW}$ receding) about the photometric minor axis. We derived the rotation curve by first subtracting the PN systemic velocity $\left(550 \mathrm{~km} \mathrm{~s}^{-1}\right)$ from the velocities and then folding and reflecting the $\mathrm{SW} P N$ velocity versus minor axis distance diagram onto the NE plot. To avoid smearing due to projection, we selected the nebulae which appear near the major axis and then binned the nebulae into groups of 10 . The average velocity in each bin and the dispersion about the mean yielded the rotation and velocity dispersion curves shown in Figure 3. The rotation curve rises from the center of the galaxy and then flattens to a value of $\sim 100 \mathrm{~km} \mathrm{~s}^{-1}$ between $10 \mathrm{kpc}$ and $20 \mathrm{kpc}$. The velocity dispersion appears to decline in the halo at distances beyond $10 \mathrm{kpc}$.
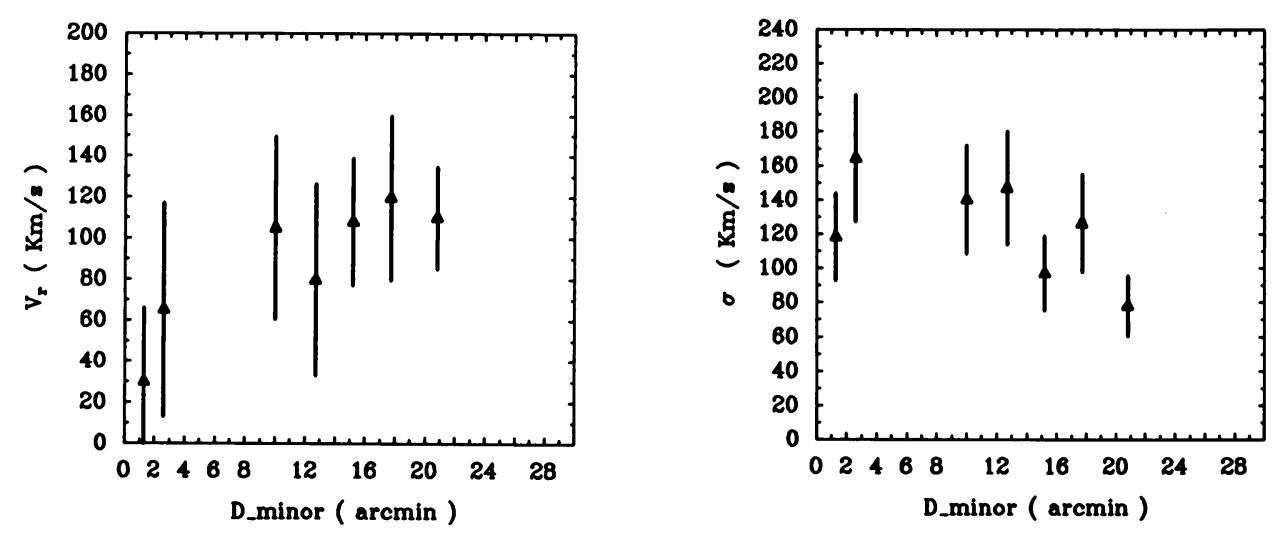

Figure 3. The left hand panel shows the observed rotation in the halo of NGC 5128 as a function of the distance from the photometric minor axis. The right hand panel shows the line-of-sight velocity dispersion in the halo. The error bars show the standard error.

We derived a preliminary mass distribution and M/L by approximating NGC 5128 as spherical and using the first moment of the collisionless Boltzmann equation (Hartwick and Sargent 1978). The rotational velocity was derived from a third order curve through Figure 3. The logarithmic derivative of the velocity dispersion was taken from a second order fit to the data in Figure 3, and included Wilkinson et al.'s central velocity dispersion of $145 \mathrm{~km} \mathrm{~s}^{-1}$. Inclusion of this point forces a relatively constant dispersion curve in the central $10 \mathrm{kpc}$, and is consistent with an isothermal core in NGC 5128. The logarithmic gradient of the space density of PN was derived from Young's (1976) asymptotic expansion for a spherical de Vaucouleur's galaxy. Finally, the blue light was integrated assuming a spherical galaxy 
following Dufour et al.'s major axis light distribution and corrected for foreground extinction with Dufour's color excess. The results from this preliminary analysis are given in Table 3 for a provisional distance of $3.3 \mathrm{Mpc}$ and the assumption that the velocity dispersion is isotropic. This analysis suggests that $\mathrm{M} / \mathrm{L}$ increases throughout the galaxy. If the plane of rotation in NGC 5128 is appreciably inclined to the line of sight, or if the velocity dispersion is anisotropic, the masses and $M / L s$ in Table 3 are lower limits to the true values. These quantities will be better constrained with more data and a more sophisticated analysis.

Table 3. Preliminary Mass Distribution and M/L in NGC 5128

\begin{tabular}{cccccc}
\hline \hline$R(k p c)$ & $V_{\text {rot }}$ & $\sigma_{V}$ & $M\left(10^{11} M_{\odot}\right)$ & $L_{B}\left(10^{10} L_{\odot}\right)$ & $M / L_{B}$ \\
\hline 1.3 & 28 & 146 & 0.2 & 0.7 & 2.4 \\
2.6 & 50 & 147 & 0.4 & 1.1 & 3.1 \\
10.0 & 104 & 137 & 1.9 & 2.4 & 7.8 \\
12.7 & 105 & 128 & 2.4 & 2.6 & 9.0 \\
15.2 & 104 & 116 & 2.7 & 2.8 & 9.8 \\
17.7 & 105 & 102 & 2.9 & 2.9 & 10.2 \\
20.8 & 113 & 81 & 3.0 & 3.0 & 10.1 \\
\hline
\end{tabular}

\section{PLANETARY NEBULAE AS STANDARD CANDLES}

If we consider two stellar populations with similar ages, chemical compositions, and mass functions, stellar evolution should drive their stars to produce planetary nebulae with similar shell masses and central star masses. For a given age of the population there will be a well determined main sequence turnoff mass and mass of the planetary central star. For a given central star mass there will be a corresponding upper limit to the ionizing flux from that star and a maximum $[O$ III] $\lambda 5007$ flux which can be produced by the nebula. Thus, we expect two similar populations to have nearly identical [O III] $\lambda 5007$ luminosity functions which are guillotined by stellar evolution at the same upper limit. Jacoby and Lesser (1981) and Ford (1983) presented data which showed that the dispersion in the reddening corrected [O III] $\lambda 5007$ magnitudes of the brightest planetary nebulae in local group galaxies is $\sim 25 \%$. This relative constancy of the maximum [O III] $\lambda 5007$ luminosity in galaxies with a wide range in mass, chemical composition, and age is due to the fact that the strong dependence of the central star's evolutionary time on its mass results in an extremely high probability of observing central stars with a very narrow dispersion about a mean mass (Shaw 1988; however, see Pottasch 1988). These observations and arguments suggest that planetaries may be good standard candles. In particular, it is reasonable to suppose that a comparison of similar populations, such as the halo of NGC 5128 and Virgo ellipticals, will lead to even smaller dispersions, and perhaps accurate relative distances.

The best way to test distances derived from planetary nebula luminosity functions (PNLFs) would be to compare the PNLF distances to independently mea- 
sured distances in the same galaxy or group of galaxies. Unfortunately, distances to even the nearest galaxies, especially early type galaxies, are so poorly determined we must at present resort to consistency checks. We first consider whether or not the PNLF appears to have the same form in different galaxies. In Figure 4 we show $\mathrm{N}(\mathrm{m})$ versus $\mathrm{m}([\mathrm{O} \mathrm{III}])$ for M81 and NGC 5128. The two histograms are nearly identical, both rising steeply to a maximum $\sim 1 \mathrm{mag}$ below the brightest nebula. Because our estimated completeness limit is only another 0.6 mags below the apparent peak, we think more observations and additional analysis of the completeness limit is needed before we can be certain that the peak is an intrinsic characteristic of the luminosity function. Jacoby's (1980) PNLF for the Magellanic Clouds does not show a turnover; however, there are too few planetaries in the first two magnitudes of the Clouds' PNLF to resolve such a feature.

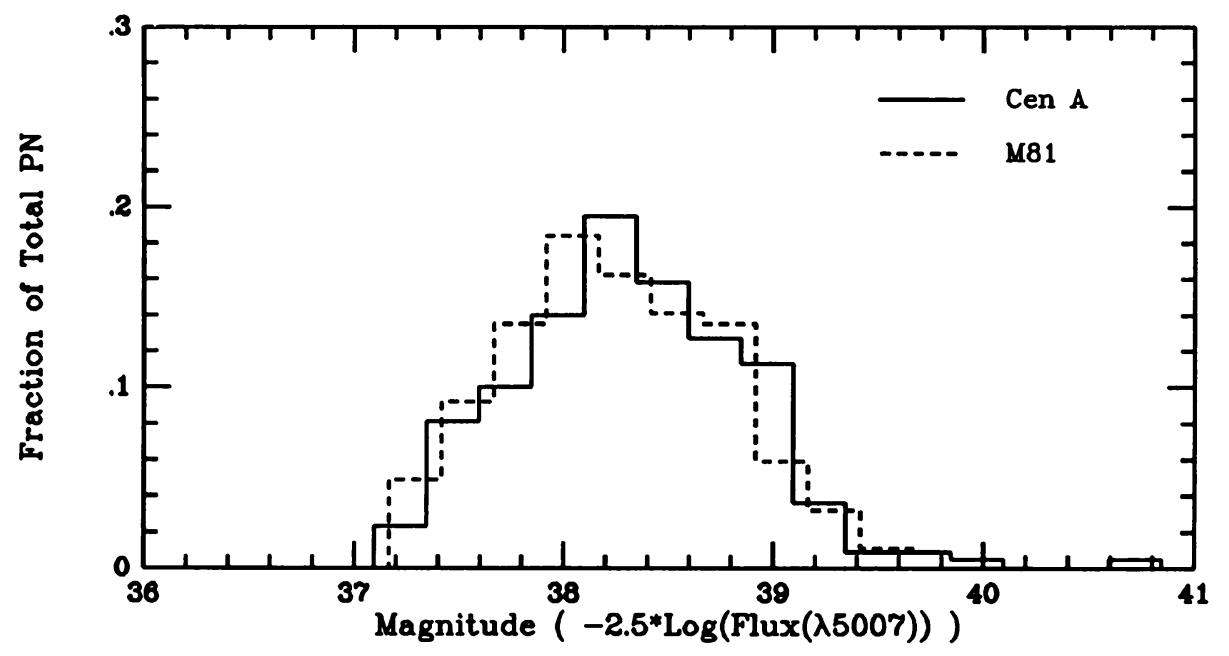

Figure 4. The planetary nebula magnitude density $\mathrm{N}(\mathrm{m})$ versus $\mathrm{m}([\mathrm{O}$ III] ג5007) for M81 and Cen A. The number of PN in each magnitude bin has been divided by the total number of nebulae.

Figure 5 shows $\log (\mathrm{N}(\mathrm{m}))$ versus $\mathrm{m}([\mathrm{O} \mathrm{III}])$ for M81, NGC 5128 , and the three galaxies in the Leo Group. All of the logarithmic PNLFs exhibit the same shape over the first magnitude, a range where we think incompleteness is not a problem. Note that the shape is not a straight line (ie, the fall-off in the distribution is not a power law). This is very important since it means the data can be fit to its unique shape. If the PNLF were a power law, the results could always be scaled by assuming a larger galaxy; however, the observed curvature in the PNLF allows it to be used directly as a standard candle.

A second consistency test can be made by comparing the relative distances of different fields within galaxies and the relative distances of galaxies within the same group. The former shows the effects of photometric errors, variable internal extinction, and statistical fluctuations in the finite manifestation of the luminosity function. The latter provides a powerful test of the constancy of the 


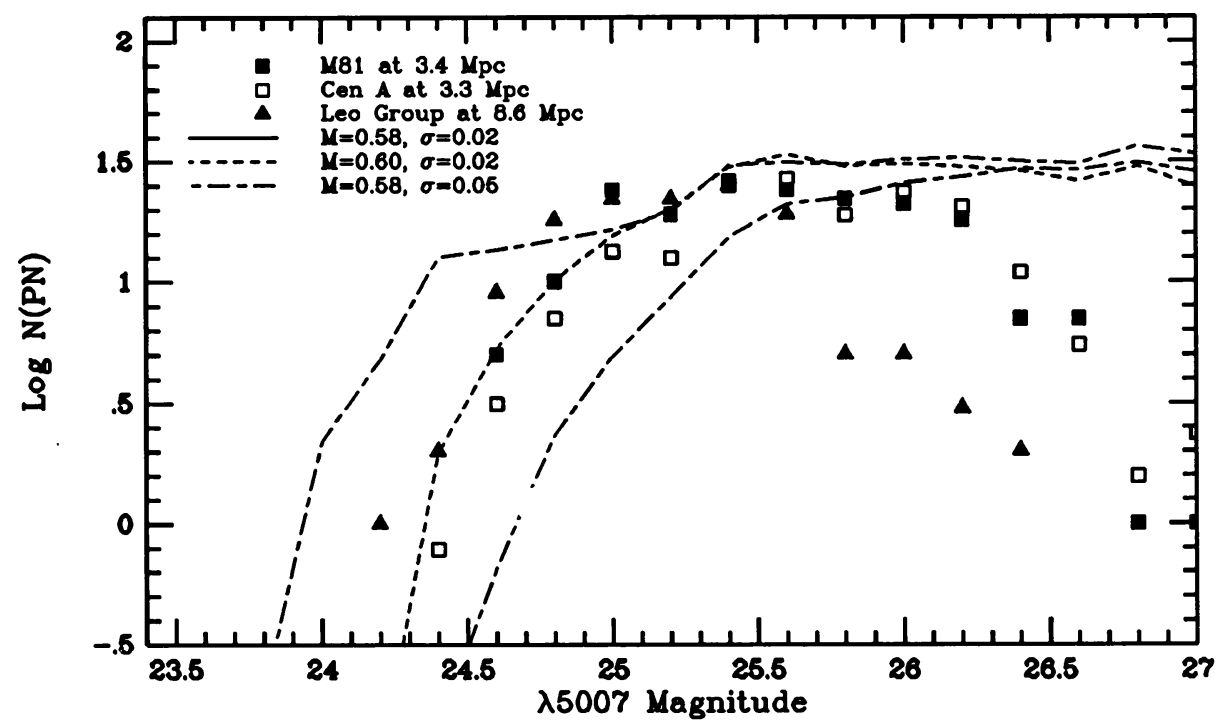

Figure 5. Logarithm of the planetary nebula number density $N(m)$ versus [O III] $\lambda 5007$ magnitude for M81, Cen A, and the sum of three galaxies in the Leo Group galaxies, corrected by the distances shown in the figure. The lines are theoretical luminosity functions for PN distributed uniformly in age, but with the Gaussian mass distribution described in the text (see §5).

PNLF in galaxies with different luminosities and Hubble types. A final goal of the consistency tests is to determine the most accurate and stable way to use the PNLF to derive distances.

There are at least three ways the PNLF can be used to derive distances: i) from the $j^{\text {th }}$ brightest nebula, ii) from a statistic such as the median derived from some fraction of the PNLF, and iii) fitting to a feature in the PNLF such as a peak or the curvature seen in Figure 5. The brightest nebula always must be treated with caution because of the possibility of confusion with faint supernova remnants or faint H II regions (in galaxies with star formation). The relative statistical merits of $m_{j}$ and $m(m e d)$ can be illustrated by supposing that the PNLF $n(m)$ versus $m$ is linear between the brightest nebula $m_{1}$ and $m_{2}$ (approximately 0.75 mag fainter than $m_{1}$ ), as is the case in NGC 5128 and M81. If

$$
n(m)=a \times\left(m-m_{1}\right) \quad m_{1}<m<m_{2}
$$

the total number of nebulae between $m_{1}$ and $m_{2}$ is

$$
N(\text { total })=(a / 2) \times\left(m_{2}-m_{1}\right)^{2} .
$$

The magnitudes of the $j^{\text {th }}$ nebula and the median nebula are

$$
\begin{aligned}
m_{j} & =m_{1}+\left(m_{2}-m_{1}\right) \times \sqrt{j / N(\text { total })} \\
m(m e d) & =m_{1}+\left(m_{2}-m_{1}\right) / \sqrt{2} .
\end{aligned}
$$


For a fixed magnitude range $m_{2}-m_{1}$ in either the program or reference galaxy, $m_{j}$ depends on $N($ total $)$ whereas the median $m(m e d)$ does not. Simply put, as the number of nebulae in $m_{2}-m_{1}$ increases, $m_{j}$ is more likely to be found close to the bright limit. The error in $m(m e d)$ is $1 / 3$ the error in $m_{1}$. Consequently, if we can confidently set $m_{2}$ above the completeness limit, $m(m e d)$ should be a better estimator than $m_{1}$ or $m_{j}$.

The (possible) peak and the curvature in the PNLF are potentially excellent standard candles. However, the magnitude of the peak (and $m(m e d)$ ) will artificially brighten and the curvature will change if the survey includes regions where a bright background or internal extinction moves the completeness limit above the true peak. Pushing the completeness limit deep enough to use either a peak or the shape of the PNLF will be very difficult for galaxies in the Virgo cluster.

Preliminary reddening corrected [O III] $\lambda 5007$ fluxes for the $1^{\text {st }}, 3^{\text {rd }}, 5^{\text {th }}$, and median nebula in the first $0.75 \mathrm{mag}$ of the luminosity function are given in Table 4. The last column gives the number of nebulae in the first 0.75 mag of the luminosity function. Distances for each galaxy or field are given relative to a provisionally adopted distance of 3.3 Mpc to NGC 5128. The distances will be revised when the final zero point is set from the PNLF in M31.

The relative distances in Table 4 show that the derived distances have a high degree of internal consistency and are relatively insensitive to the distance estimator. The median [O III] $\lambda 5007$ flux appears to give somewhat more consistent results than the $j^{t h}$ brightest nebula. The high degree of internal consistency for the three galaxies in the Leo Group $(8.63 \mathrm{Mpc}, \sigma=0.14 \mathrm{Mpc})$ is particularly encouraging.

The distance estimates to NGC 4486 were made after eliminating the brightest nebula, which was 1.48 times brighter than the second brightest nebula. Neither any of the other galaxies nor any of the fields within the galaxies had such a large difference between the two brightest candidates. The $\mathrm{H} \alpha$ exposures of NGC 4486 (cf Table 1) did not show any nebulae at the positions of the PN candidates. Although there is little basis for guessing what the spectrum of a Type I SNR in the halo of NGC 4486 might look like, in most known SNRs $\mathrm{H} \alpha$ is stronger than [O III] $\lambda 5007$ (eg. D'Odorico and Dopita 1983). The exceptions are oxygen rich Type IIs (Tuohy and Dopita 1983) in which the large expansion velocities would overfill our $30 \AA \lambda 5007$ filter. Thus, even in these unlikely SNRs $\mathrm{H} \alpha$ probably would be as bright or brighter than [O III] $\lambda 5007$. Although we conclude that the majority of our nebulae are not SNRs, we think the PNLF-based distance of M87 relative to the other galaxies should be viewed as highly provisional until we are able to confirm that the faint candidates in M87 are indeed planetary nebulae.

Possible sources of errors such as confusion, bandpass-redshift alignment and filter calibration, and internal reddening will be discussed in more detail in subsequent papers. We note here that the uncertainty in the magnitudes due to these difficulties can be eliminated by obtaining moderate dispersion spectra of the brightest nebulae in each galaxy. We plan to undertake this important and difficult task.

In conclusion, we think the observations presented in this review strongly support our contention the the PNLF will prove to be an accurate and reliable 
Table 4. [O III] $\lambda 5007$ Fluxes for the Brightest Planetary Nebulae in the Survey Galaxies

\begin{tabular}{|c|c|c|c|c|c|}
\hline \multirow{3}{*}{$\begin{array}{l}\text { Galaxy } \\
5128 \text { (All) }\end{array}$} & \multicolumn{5}{|c|}{$\begin{array}{l}{\left[\mathrm{O} \mathrm{III]} \lambda 5007 \text { Fluxes }\left(\operatorname{ergs~} \mathrm{cm}^{-2} \mathrm{~s}^{-1} \times 10^{-15}\right)\right.} \\
\text { and Provisional Distances }(\mathrm{Mpc}) \text { Relative to NGC } 5128\end{array}$} \\
\hline & \multirow{2}{*}{$\begin{array}{c}1^{\text {st }} \\
1.45\end{array}$} & \multirow{2}{*}{$\begin{array}{c}3^{\text {rd }} \\
1.26\end{array}$} & \multirow{2}{*}{$\begin{array}{c}5^{t h} \\
1.23\end{array}$} & \multicolumn{2}{|c|}{$\begin{array}{l}\text { median } N(\text { medi } \\
\text { (First } 0.75 \text { mag })\end{array}$} \\
\hline & & & & 0.918 & 46 \\
\hline & 3.3 & 3.3 & 3.3 & 3.3 & \\
\hline \multirow[t]{2}{*}{$5128(\mathrm{SW})$} & 1.03 & 0.897 & 0.773 & 0.827 & 7 \\
\hline & 3.92 & 3.91 & 4.16 & 3.48 & \\
\hline \multirow[t]{2}{*}{$5128(\mathrm{NE})$} & 1.26 & 1.15 & 1.04 & 0.945 & 18 \\
\hline & 3.54 & 3.45 & 3.59 & 3.25 & \\
\hline \multirow[t]{2}{*}{5128 (Envl) } & 1.45 & 1.25 & 1.04 & 0.929 & 18 \\
\hline & 3.30 & 3.31 & 3.59 & 3.28 & \\
\hline \multirow[t]{2}{*}{ M81 (All) } & 1.37 & 1.29 & 1.21 & 0.862 & 52 \\
\hline & 3.39 & 3.26 & 3.32 & 3.40 & \\
\hline \multirow[t]{2}{*}{3115 (All) } & 0.276 & 0.254 & 0.225 & 0.175 & 21 \\
\hline & 7.56 & 7.35 & 7.72 & 7.56 & \\
\hline \multirow[t]{2}{*}{$3115(E)$} & 0.254 & 0.179 & 0.159 & 0.141 & 14 \\
\hline & 7.88 & 8.76 & 9.18 & 8.42 & \\
\hline \multirow[t]{2}{*}{$3115(W)$} & 0.276 & 0.247 & 0.186 & 0.175 & 15 \\
\hline & 7.56 & 7.45 & 8.49 & 7.56 & \\
\hline \multicolumn{6}{|l|}{ Leo Group } \\
\hline \multirow[t]{2}{*}{3377} & 0.200 & 0.169 & 0.156 & 0.129 & 13 \\
\hline & 8.89 & 8.98 & 9.27 & 8.80 & \\
\hline \multirow[t]{2}{*}{3384 (All) } & 0.202 & 0.187 & 0.174 & 0.137 & 25 \\
\hline & 8.84 & 8.58 & 8.77 & 8.54 & \\
\hline \multirow[t]{2}{*}{$3384(E)$} & 0.197 & 0.174 & 0.155 & 0.137 & 15 \\
\hline & 8.95 & 8.88 & 9.30 & 8.54 & \\
\hline \multirow[t]{2}{*}{$3384(W)$} & 0.202 & 0.154 & 0.151 & 0.146 & 16 \\
\hline & 8.84 & 9.44 & 9.42 & 8.27 & \\
\hline \multirow[t]{2}{*}{3379 (All) } & 0.196 & 0.187 & 0.175 & 0.136 & 24 \\
\hline & 8.98 & 8.57 & 8.75 & 8.57 & \\
\hline \multirow[t]{2}{*}{$3379(E)$} & 0.196 & 0.175 & 0.170 & 0.137 & 14 \\
\hline & 8.98 & 8.85 & 8.88 & 8.54 & \\
\hline \multirow[t]{2}{*}{$3379(W)$} & 0.187 & 0.146 & 0.139 & 0.135 & 10 \\
\hline & 9.19 & 9.69 & 9.82 & 8.61 & \\
\hline \multirow[t]{2}{*}{5866 (All) } & 0.177 & 0.123 & 0.113 & 0.113 & 10 \\
\hline & 9.45 & 10.56 & 10.89 & 9.43 & \\
\hline \multirow[t]{2}{*}{4486 (All) } & 0.100 & 0.081 & 0.065 & 0.063 & 10 \\
\hline & 12.57 & 13.06 & 14.35 & 12.59 & \\
\hline
\end{tabular}


way to estimate the distances of early type galaxies out to the distance of the Virgo cluster.

\section{IMPLICATIONS FOR PLANETARY NEBULA CENTRAL STARS}

The [O III] flux in planetary nebulae is driven by the ionizing UV radiation emerging from the central star. If the central star passes through its maximum luminosity and temperature while the nebula is still optically thick to ionizing radiation, the nebula can attain a high luminosity. If the nebula expands more rapidly than the central star can evolve across to the blue side of the HR diagram (low mass central stars), only a small fraction of the nebula is ionized and its luminosity will be low. If the central star evolves very quickly (high mass central stars), the lifetime of the high luminosity period may be so short that very few, if any, will be seen.

To generate a theoretical planetary nebula luminosity function we began with Wood and Faulkner's (1986) models for the evolution of central stars. We ionized model nebulae with a series of energy distributions corresponding to ages along the evolutionary track of the central star. The $0.6 M_{\odot}$ nebulae were evolved by allowing them to expand uniformly at a constant rate. The models show that the precise value of the nebular mass is unimportant because only a small fraction of the total mass is ionized in the brightest nebulae.

The following simplifying assumptions were made to expedite computation of the models: 1) the nebula is a uniform density sphere, 2) the nebula expands at a constant velocity of $\left.20 \mathrm{~km} \mathrm{~s}^{-1}, 3\right)$ the nebula is always in ionization equilibrium with the UV flux from the central star, 4) no additional energy input is available (eg. there is no stellar wind), 5) the central star flux is a black body energy distribution, 6) the central star masses have a Gaussian distribution, and 7) the chemical composition is typical of solar neighborhood nebulae (Aller and Czyzak 1983).

A series of masses of $0.6,0.7$, and $0.76 M_{\odot}$ were chosen from the evolutionary tracks presented in Figure $1 \mathrm{~b}$ of Wood and Faulkner (1986), and a mass of 0.65 was interpolated from this figure. A mass track at 0.565 was also taken from Figure 2 of Schönberner (1983). This track is not entirely consistent with those of Wood and Faulkner, but is adequate for the fainter nebulae, and has little bearing on our principle interest-the high luminosity nebulae.

Wood and Faulkner present four sequences of tracks corresponding to two mass loss scenarios and two epochs for PN shell ejection. We chose the mass loss model in which PNN lose mass at a rate of $3 \times 10^{-5} M_{\odot} \mathrm{yr}^{-1}$ until $\log T_{\text {eff }}=3.8$, after which time $\dot{M}$ is zero. We selected the shell ejection epoch at the rising surface luminosity of a flash rather than midway between helium shell flashes. These choices were invoked to provide a set of models having the lowest UV fluxes. A set having higher fluxes requires smaller central star masses.

For each central star mass, the temperature and luminosity were interpolated into a grid spaced at 1000 year intervals from the moment the central star reaches $\log T_{\text {eff }}=4.0$. Black body energy distributions for central stars at these ages were used as ionizing sources in the model nebula code described by Aller and 
Keyes (1980), based on the formulation by Balick (1975).

The same nebula was used in each mass sequence, and the age of the nebula determined the size and density. Note that the age includes an offset on the order of several hundred years relative to the central star age zero point, since there is a delay from planetary nebula ejection until the central star reaches $\log T_{\text {eff }}=4.0$.

The [O III] $\lambda 5007$ intensities predicted by the models were used to generate a grid of fluxes as a function of age and central star mass. We then randomly selected planetaries in a Monte Carlo simulation of observing many extragalactic PN according to the following prescriptions: 1) PN are distributed uniformly in age from 0 to 30,000 years, and 2) $P N$ central stars have a Gaussian distribution such that the number of stars of mass $M$ is proportional to $\exp \left(-0.5 \times(M / \sigma)^{2}\right)$. We then randomly selected $100,000 \mathrm{PN}$ ages and masses, interpolated in the [O III] $\lambda 5007$ luminosity grid, and histogramed the results.

Figure 5 illustrates three such models for mass distributions selected with peaks at $0.58,0.60$, and $0.58 M_{\odot}$ and $\sigma(M)$ of $0.02,0.02$, and $0.05 M_{\odot}$. The curves in this figure have been scaled vertically to match the observed number of $P N$ in M81. The horizontal magnitude scale is simply $-2.5 \times \log F([O I I I] \lambda 5007)-10$ for nebulae at a distance of $1 \mathrm{Mpc}$. The principle features in the curves are: 1) a sharp cutoff at the bright end, 2) a flat distribution beginning at $\sim 1$ mag below the brightest, 3) a bright end shift of about $0.3 \mathrm{mag}$ for a mass shift of $0.02 M_{\odot}$, and 4) a bright end tail of about $0.7 \mathrm{mag}$ when the mass function is broadened to $0.05 M_{\odot}$. A comparison with the data for M81, Cen A (NGC 5128), and the three galaxies in Leo shows that the cutoff at the high mass end of the central star mass distribution must be quite abrupt.

It is important to note that the bright end cutoff is completely controlled by the high mass tail of the central star mass distribution. A model which has a long tail of low mass central stars does not change the shape of the cutoff.

We conclude that, within the limits of the models, the masses of central stars peak near $0.6 M_{\odot}$, and have a high mass tail characterized by a Gaussian distribution with $\sigma(M)<0.05 M_{\odot}$, and probably closer to $0.02 M_{\odot}$. For comparison, the observationally selected distribution derived by Schönberner (1981) and Schönberner and Weidemann (1983) suggests a central star mass peak near $0.58 M_{\odot}$, and an equivalent $\sigma(M) \approx 0.015 M_{\odot}$. We caution the reader that the assumptions in the models may have a significant impact on the upper limits to the [O III] $\lambda 5007$ luminosities, but the shape of the luminosity distributions will not be altered much since these are dominated by the central star mass distribution.

This work was supported by NASA contracts NAS 5-29293 and NAGW-421. Don Neill provided excellent help in analysis of the NGC 5128 observations. We are grateful to C.D. Keyes for providing the nebula model code and instructions on how to use it. 


\section{REFERENCES}

Aller, L.H. and Czyzak, S.J. 1983, Ap. J. Suppl., 51, 211.

Aller, L.H. and Keyes, C.D. 1980, Ap. Sp. Sci., 72, 203.

Balick, B. 1975, Ap. J., 203, 705.

Cahn, J. H. and Wyatt, S. P. 1978, in IAU Symposium 76, Planetary Nebulae Observations and Theory, ed. Y. Terzian (Dordrecht:Reidel), p. 3.

Cudworth, K. M. 1974, A. J., 79, 1384.

Dopita, M. A., Meatheringham, S. J., Wood, P. R., Ford, H. C., Webster, B. L., and Morgan, D. H. 1988, Paper presented at the ESO Workshop on "The Evolution and Dynamics of the Outer Halo of the Galaxy" April 7-9, 1987.

D'Odorico, S. and Dopita, M. 1983, in IAU Symposium 101, Supernova Remnants and Their $X$-ray Emission, ed. J. Danziger and P. Gorenstein (Dordrecht : Reidel), p. 517.

Dufour, R. J., van den Bergh, S., Harvel, C. A., Martins, D. H., Schiffer, III, F. H., Talbot, Jr., R. J., Talent, D. L., and Wells, D. N. 1979, A. J., 84, 284.

Eather, R. H. and Reasoner, D. L. 1969, Appl. Optics, 8, 227.

Ford, H.C. 1983, in IAU Symposium 103, Planetary Nebulae, ed. D.R. Flower (Dordrecht : Reidel), p. 443.

Ford, H. C., Freeman, K. C., Hui, X., Ciardullo, R., Dopita, M. A., Jacoby, G., and Meatheringham, S. 1988, in preparation.

Keyes, C.D. and Aller, L.H. 1980, Ap. Sp. Sci., 72, 203.

Hartwick, F. D. A. and Sargent, W. L. W. 1978, Ap. J., $221,512$.

Huchra, J., Stauffer, J., and van Speybroeck, L. 1982, Ap. J. (Letters), 259 , L57.

Jacoby, G. H. 1980, Ap. J. Suppl., 42, 1.

Jacoby, G. and Ford, H. C. 1986, Ap. J., 304, 490.

Jacoby, G. and Ford, H. C. 1988, in preparation.

Jacoby, G. H. and Lesser, M. P. 1981, A. J., 86, 185.

Jaffe, W. 1983, M.N.R.A.S., 202, 995.

Lawrie, D. G. 1983, Ap. J., $273,562$.

Lawrie, D. G. and Ford, H. C. 1982, Ap. J., 256, 120.

Merritt, D. 1985, A. J., 90, 1027.

Nolthenius, R. 1984, Ph.D. thesis, University of California, Los Angeles.

Nolthenius, R. and Ford, H. C. 1986, Ap. J., 305, 600.

Nolthenius, R. and Ford, H. C. 1987, Ap. J., 317, 62.

Pottasch, S. R. 1988, IAU Symposium 191, ed. S. Torres-Peimbert (Dordrecht : Reidel), this volume.

Schönberner, D. 1981, Astr. Ap, 103, 119.

Schönberner, D. 1983, Ap. J., $272,708$.

Schönberner, D. and Weidemann, V. 1983, IAU Symposium 103, Planetary Nebulae, ed. D.R. Flower (Dordrecht : Reidel), p. 359.

Shaw, R. A. 1988, IAU Symposium 191, ed. S. Torres-Peimbert (Dordrecht : Reidel), this volume.

Tonry, J. L. 1984, Ap. J. (Letters), 283, L27. 
Tuohy, I. R. and Dopita, M. A. 1983, in IAU Symposium 101, Supernova Remnants and Their $X$-ray Emission, ed. J. Danziger and P. Gorenstein (Dordrecht : Reidel) p. 165.

van den Bergh, S. 1969, Ap. J. Suppl., 19, 145.

Whitmore, B. C. 1980, Ap. J., $242,53$.

Wilkinson, A., Sharples, R. M., Fosbury, R. A. E., and Wallace, P. T. 1986, M.N.R.A.S., 218, 297.

Wood, P.R. and Faulkner, D.J. 1986, Ap. J., 307, 659.

Young, P. J. 1976, A. J., 81, 807. 